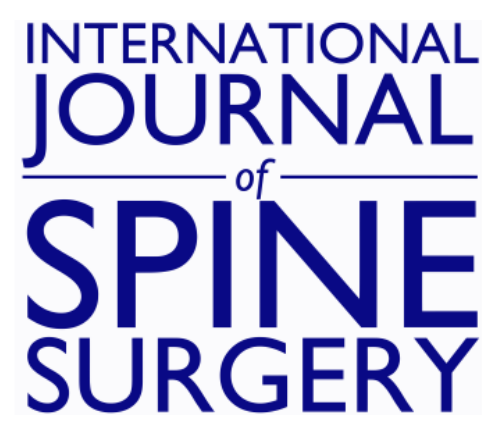

\title{
Impact of Depression Severity on Patient-Reported Outcome Measures Following Multilevel Anterior Cervical Discectomy and Fusion
}

Elliot D.K. Cha, Conor P. Lynch, Caroline N. Jadczak, Shruthi Mohan, Cara E. Geoghegan and Kern Singh

Int J Spine Surg published online 17 February 2022

http://ijssurgery.com/content/early/2022/03/01/8180

This information is current as of April 25, 2023.

Email Alerts Receive free email-alerts when new articles cite this article. Sign up at: http://ijssurgery.com/alerts

The International Journal of Spine Surgery

2397 Waterbury Circle, Suite 1,

Aurora, IL 60504, Phone: +1-630-375-1432 


\title{
Impact of Depression Severity on Patient-Reported Outcome Measures Following Multilevel Anterior Cervical Discectomy and Fusion
}

\author{
ELLIOT D.K. CHA, MS ${ }^{1}$; CONOR P. LYNCH, MS ${ }^{1}$; CAROLINE N. JADCZAK, BS ${ }^{1}$; SHRUTHI MOHAN, BS ${ }^{1}$; \\ CARA E. GEOGHEGAN, BS ${ }^{1}$; AND KERN SINGH, MD ${ }^{1}$ \\ ${ }^{I}$ Department of Orthopaedic Surgery, Rush University Medical Center, Chicago, IL, USA
}

\begin{abstract}
Background: Few studies have investigated the effects of preoperative depression and multilevel procedures on patientreported outcomes (PROs) following anterior cervical discectomy and fusion (ACDF). This study aims to determine the impact of preoperative depression on PROs in single vs multilevel ACDF procedures.

Methods: Eligible primary single or multilevel ACDF procedures were retrospectively reviewed from 2015 to 2020 using a surgical database. PROs included visual analog scale (VAS), Neck Disability Index (NDI), 12-Item Short Form Physical Composite Summary and Mental Composite Summary (SF-12 PCS and MCS), and Patient-Reported Outcomes Measurement Information System physical function (PROMIS PF). PROs were collected preoperatively and at 6 weeks, 12 weeks, 6 months, and 1 year postoperatively. Patients were stratified into 3 groups based on Patient Health Questionnaire-9 (PHQ-9) score. Differences in PROs were assessed based on preoperative depression severity or both preoperative depression severity and number of operative levels.

Results: Our patient cohort included 42 individuals with minimal preoperative depressive symptoms, 32 having mild and 27 having moderate to severe. Baseline PRO values were significantly different between groups (all $P<0.01$ ). Improvement significantly differed between groups at 6 weeks for VAS arm, NDI, and SF-12 MCS (all $P<0.05$ ), and at 12 wweeks for VAS neck, NDI, SF-12 PCS and MCS, and PROMIS PF (all $P<0.05$ ). SF-12 PCS and MCS at 6 months and VAS arm and SF-12 MCS at 1 year demonstrated significant intergroup differences (all $P<0.05)$. VAS arm at 1 year $(P=0.029)$, NDI at 12 weeks $(P$ $=0.034)$, PROMIS PF at 6 weeks $(P=0.038)$, and SF-MCS at all postoperative time points were impacted by both preoperative depression severity and number of levels fused.

Conclusion: Depression severity impacted recovery of pain, disability, and physical function preoperatively and at intermittent postoperative time points. Both severity and multilevel procedures impacted pain and mental health at intermittent postoperative time points. In addition to depression, multilevel ACDF procedures are an additional factor that must be considered in expected improvement of postoperative outcomes.

Level of Evidence: 4.

Clinical Relevance: Multilevel ACDF procedures and preoperative depression severity both impact postoperative pain, disability, and physical function.
\end{abstract}

Cervical Spine

Keywords: cervical fusion, PROMIS, patient-reported outcome measures (PROMs), depression, PHQ-9

\section{INTRODUCTION}

Depression is one of the most common mental disorders globally, with more than 250 million suffering from the disease, and it is considered one of the leading causes of disability worldwide. ${ }^{1}$ With how commonplace this disorder is becoming, it is increasingly important for clinicians to consider a patient's mental health as a contributing factor toward their overall health. One method to keep track of a patient's overall mental well-being is through the use of patient-reported outcome measures (PROMs), which gauges their perception of various aspects of their health status.
One such PROM to assess depression is the Patient Health Questionnaire-9 (PHQ-9). This questionnaire is a reliable and accurate screening tool for major depressive disorder and is based on criteria outlined in the Diagnostic and Statistical Manual of Mental Disorders (DSM-5). ${ }^{2}$ Although a self-reported questionnaire, PHQ-9 has been established as a useful diagnostic tool for clinicians and has been validated in both lumbar and cervical spine patient populations. ${ }^{3-5}$ Its application has been especially useful among cervical radiculopathy patients presenting with pain and disability; both of which are proven risk factors for depression. ${ }^{6,7}$ Anterior cervical discectomy and fusion 
$(\mathrm{ACDF})$ is a proven treatment for a radiculopathy and has demonstrated favorable outcomes and high satisfaction ratings. ${ }^{8,9}$ However, when considering the impact of depression on postoperative improvements, current studies indicate less favorable outcomes. ${ }^{10}$

ACDF patients preoperatively diagnosed with depression are at an increased risk of adverse outcomes, increased opioid consumption, and increased healthcare expenses compared to those without depression. ${ }^{11}$ While an overwhelming majority of studies outline a strong association between preoperative depression and worse overall outcomes in pain and disability following single-level ACDF procedures, ${ }^{6,12}$ few report on the effects of depression on multilevel procedures. Such procedures have been demonstrated to have higher instances of complications, increased rates of revisions, and worse postoperative outcomes with the increasing number of operative levels. ${ }^{13,14}$ Coupled with the negative effects of depression on postoperative outcomes, it is possible that depressed individuals undergoing multilevel procedures may be at even greater risk for poorer outcomes and ultimately lower health-related quality of life. Currently, there is a dearth of information surrounding depression and its effects on outcomes in multilevel ACDF procedures, and no study to date has focused on depression severity and its impact on these specific procedures. Therefore, the study aims to focus on the impact that multilevel procedures have on outcomes among individuals with varying levels of depression to help clinicians better counsel patients preoperatively. We hypothesize that patients will demonstrate worse pain, disability, and mental health following a multilevel ACDF as compared to singlelevel procedures.

\section{METHODS}

\section{Patient Population}

In accordance with our institution's ethical guidelines, Institutional Review Board approval (ORA 14051301) and patient-informed consent were obtained for this study. A retrospective review of a surgical database was performed to identify eligible cervical spine procedures from March 2015 to January 2020. All procedures were performed by a single attending physician at a single institution. Inclusion criteria were defined as adult patients who underwent a primary, elective, single, or multilevel ACDF for degenerative spinal pathology. Exclusion criteria were defined as patients undergoing a primary or revision procedure for treatment of an infection, malignancy, or trauma. Initial screening of the surgical registry identified 305 eligible patients. After inclusion and exclusion criteria were applied the final study cohort consisted of 101 patients.

\section{Data Collection}

Information pertaining to patients' age, sex, body mass index, smoker status, ethnicity, and diabetic status were collected as the part of their demographics. Physical classification and comorbidity burden were also recorded as American Society of Anesthesiologists classification and Charlson Comorbidity Index, respectively. Spinal pathologies were recorded along with perioperative characteristics, which included operative duration (skin incision to skin closure), estimated blood loss, length of postoperative stay, and day of discharge.

The primary outcomes of interest were PROMs for pain, disability, physical function, and mental health. Neck and arm pain were evaluated using the visual analog scale (VAS) whereas disability was assessed using the Neck Disability Index (NDI). Physical function was evaluated using 2 separate PROMs, either 12-Item Short Form Health Survey Physical Component Score (SF-12 PCS) or Patient-Reported Outcome Measurement Information System physical function (PROMIS PF). Lastly, mental health was evaluated using both the PHQ-9 as well as the 12-Item Short Form Mental Composite Score (SF-12 MCS). All PROMs were collected at the preoperative time point and subsequently at the 6-week, 12-week, 6-month, and 1-y postoperative time points.

\section{Statistical Analysis}

All statistical analysis was performed using StataIC 16.1 (StataCorp, College Station, TX, USA). Prior to analysis, patients were categorized into 3 groups based on preoperative PHQ-9: minimal $(<5)$, mild (5-9), and moderate to severe $(>9)$. Descriptive statistics were performed for demographic and perioperative characteristics as well as for all PROMs. Univariate analysis was performed to evaluate significant differences, in demographics and perioperative characteristics, between depression groups using either a chi-square test for categorical variables or a two-tailed $t$ test for continuous variables. The impact of preoperative depression severity on PROM values was evaluated using a simple linear regression. In order to determine the impact of multilevel procedures in addition to severity of preoperative depression on postoperative PROMs scores, a multiple linear regression was performed. All statistical analysis required a $P \leq 0.050$ for significance. 
Table 1. Patient demographics by depression severity.

\begin{tabular}{|c|c|c|c|c|}
\hline Characteristic & $\begin{array}{c}\text { Minimal } \\
(n=42)\end{array}$ & $\begin{array}{c}\text { Mild } \\
(n=32)\end{array}$ & $\begin{array}{c}\text { Moderate/Severe } \\
(n=27)\end{array}$ & $P$ value* \\
\hline Age $($ mean $\pm S D)$ & $50.6 \pm 11.0$ & $48.6 \pm 7.7$ & $46.9 \pm 9.9$ & 0.297 \\
\hline Gender & & & & 0.897 \\
\hline Female & $42.9 \%(18)$ & $37.5 \%(12)$ & $40.7 \%(11)$ & \\
\hline Male & $57.1 \%(24)$ & $62.5 \%(20)$ & $59.3 \%(16)$ & \\
\hline BMI & & & & 0.567 \\
\hline$<30 \mathrm{~kg} / \mathrm{m}^{2}$ & $64.3 \%(27)$ & $56.3 \%(18)$ & $51.9 \%(14)$ & \\
\hline$\geq 30 \mathrm{~kg} / \mathrm{m}^{2}$ & $35.7 \%(15)$ & $43.7 \%(14)$ & $48.1 \%(13)$ & \\
\hline Smoking Status & & & & 0.895 \\
\hline Nonsmoker & $85.7 \%(36)$ & $84.4 \%(27)$ & $81.5 \%(22)$ & \\
\hline Smoker & $14.3 \%(6)$ & $15.6 \%(5)$ & $18.5 \%(5)$ & \\
\hline Ethnicity & & & & 0.028 \\
\hline White & $80.9 \%(34)$ & $81.2 \%(26)$ & $63.0 \%(17)$ & \\
\hline African American & $11.9 \%(5)$ & $6.3 \%(2)$ & $0.0 \%(0)$ & \\
\hline Hispanic & $2.4 \%(1)$ & $6.3 \%(2)$ & $25.9 \%(7)$ & \\
\hline Asian & $4.8 \%(2)$ & $3.1 \%(1)$ & $3.7 \%(1)$ & \\
\hline Other & $0.0 \%(0)$ & $3.1 \%(1)$ & $7.4 \%(2)$ & \\
\hline Diabetes & & & & 0.987 \\
\hline Diabetic & $88.1 \%(37)$ & $87.5 \%(28)$ & $88.9 \%(24)$ & \\
\hline Nondiabetic & $11.9 \%(5)$ & $12.5 \%(4)$ & $11.1 \%(3)$ & \\
\hline ASA Classification & & & & 0.159 \\
\hline$\leq 2$ & $80.4 \%(21)$ & $90.6 \%(20)$ & $95.8 \%(23)$ & \\
\hline$>2$ & $19.5 \%(8)$ & $9.4 \%(3)$ & $4.2 \%(1)$ & \\
\hline CCI & & & & 0.159 \\
\hline$\leq 1$ & $52.5 \%(33)$ & $69.0 \%(20)$ & $74.1 \%(20)$ & \\
\hline$>1$ & $47.5 \%(19)$ & $31.0 \%(9)$ & $25.9 \%(7)$ & \\
\hline
\end{tabular}

Abbreviations: ASA, American Society of Anesthesiologists; BMI, body mass index; CCI, Charlson Comorbidity Index. ${ }^{*} P$ value calculated using $\chi^{2}$ analysis or Student's $t$ test.

Boldface indicates statistical significance.

\section{RESULTS}

Of the 101 patients included in this study, 42 were categorized as having minimal depressive symptoms, 32 having mild symptoms, and 27 having moderate to severe. The study cohort had a mean age of 48.1 years with $60 \%$ being men and $59 \%$ nonobese $\left(\right.$ BMI $\left.<30 \mathrm{~kg} / \mathrm{m}^{2}\right)$. Tests for independence of demographics between groups demonstrated no significant differences except for ethnicity $(P=0.028)$ (Table 1). Majority of patients had a spinal pathology of myeloradiculopathy (88.1\%), and a higher proportion of procedures were treated at the single level (58.4\%). Mean operative time was 54.3 min with an average estimated blood loss of $29.8 \mathrm{~mL}$. No significant perioperative differences were observed between depression groups (all $P>0.100$ ) (Table 2).

\section{Primary Outcomes}

Regression analysis revealed that preoperative scores for VAS neck, VAS arm, NDI, SF-12 PCS,

Table 2. Perioperative characteristics by depression severity.

\begin{tabular}{|c|c|c|c|c|}
\hline Characteristic & $\begin{array}{c}\text { Minimal } \\
(n=42)\end{array}$ & $\begin{array}{c}\text { Mild } \\
(n=32)\end{array}$ & $\begin{array}{c}\text { Moderate/Severe } \\
(n=27)\end{array}$ & $P$ value* \\
\hline \multicolumn{5}{|l|}{ Spinal pathology } \\
\hline Herniated nucleus pulposus & $85.7 \%(36)$ & $90.6 \%(29)$ & $85.2 \%(23)$ & 0.510 \\
\hline Spinal stenosis & $57.1 \%(24)$ & $65.6 \%(21)$ & $66.7 \%(18)$ & 0.655 \\
\hline Myeloradiculopathy & $92.8 \%(39)$ & $81.2 \%(26)$ & $88.9 \%(24)$ & 0.578 \\
\hline Number of operative levels & & & & 0.590 \\
\hline Single & $64.3 \%(27)$ & $53.1 \%(17)$ & $55.6 \%(15)$ & \\
\hline Multilevel & $35.7 \%(15)$ & $46.9 \%(15)$ & $44.4 \%(12)$ & \\
\hline Operative time, min, mean $\pm \mathrm{SD}$ & $47.2 \pm 87.1$ & $63.5 \pm 19.3$ & $54.4 \pm 18.2$ & 0.489 \\
\hline Estimated blood loss, $\mathrm{mL}$, mean $\pm \mathrm{SD}$ & $31.7 \pm 14.8$ & $26.3 \pm 9.4$ & $30.8 \pm 12.9$ & 0.202 \\
\hline Length of stay, $h$, mean \pm SD & $13.0 \pm 9.9$ & $13.1 \pm 14.2$ & $12.5 \pm 15.4$ & 0.981 \\
\hline Day of discharge & & & & 0.138 \\
\hline POD0 & $71.4 \%(30)$ & $81.3 \%(26)$ & $80.0 \%(20)$ & \\
\hline POD1 & $26.1 \%(11)$ & $9.4 \%(3)$ & $16.0 \%(4)$ & \\
\hline POD2 & $2.3 \%(1)$ & $9.4 \%(3)$ & $0.0 \%(0)$ & \\
\hline POD3 & $0.0 \%(0)$ & $0.0 \%(0)$ & $4.0 \%(1)$ & \\
\hline
\end{tabular}

Abbreviation: POD, postoperative day.

${ }^{*} P$ value calculated using $\chi^{2}$ analysis or Student's $t$ test. 
PROMIS PF, and SF-12 MCS worsened based on preoperative depression severity (all $P<0.010$ ). VAS neck demonstrated significantly different postoperative scores based on preoperative depression severity at the 12 -week timepoint only $(P=0.012)$. VAS arm demonstrated significant differences between groups at the 6 -week $(P=0.031)$ and 1 -year $(P=$ $0.031)$ postoperative time points. NDI scores were significantly worse with the increasing severity of preoperative depression at the 6-week $(P=0.009)$ and 12-week $(P=0.001)$ timepoint, but this difference did not persist past the 6-month timepoint. Physical function demonstrated variable differences between the 2 metrics, SF-12 PCS and PROMIS PF. Differences in postoperative SF-12 PCS scores between groups were observed at the 12 -week $(P=$ $0.019)$ and 6-month $(P=0.038)$ timepoint only. Similarly, PROMIS PF demonstrated worse postoperative scores with more severe preoperative depressive symptoms at the 12 -week timepoint only $(P=0.046)$. Mental health demonstrated significant differences between all groups at all postoperative timepoints (all $P<0.01$ ). A summary of postoperative PROM scores as a function of worsening preoperative depression is found in Table 3.

\section{Impact of Multilevel Procedures}

The collective impact of multilevel ACDF procedures and preoperative depression severity demonstrated significant effects on postoperative scores for VAS arm, NDI, PROMIS PF, and SF-12 MCS (all $P$ $<0.05)$. More specifically, although no difference in scores due to the added effect of a multilevel procedure was demonstrated for VAS neck, VAS arm demonstrated a significant difference between groups at the

Table 3. Postoperative improvement by depression severity and number of levels fused.

\begin{tabular}{|c|c|c|c|c|c|}
\hline PROM & $\begin{array}{c}\text { Minimal } \\
\text { Mean } \pm \text { SD }(n)\end{array}$ & $\begin{array}{c}\text { Mild } \\
\text { Mean } \pm \text { SD }(n)\end{array}$ & $\begin{array}{c}\text { Moderate/Severe } \\
\text { Mean } \pm \text { SD }(n)\end{array}$ & $P$ value* & $P$ value $\dagger$ \\
\hline \multicolumn{6}{|l|}{ VAS neck } \\
\hline Preoperative & $4.7 \pm 2.4(40)$ & $6.5 \pm 2.1(30)$ & $7.4 \pm 2.3(27)$ & $<0.001$ & - \\
\hline $6 \mathrm{wk}$ & $3.0 \pm 2.6(38)$ & $3.4 \pm 2.4(30)$ & $3.6 \pm 2.7(24)$ & 0.292 & 0.789 \\
\hline 12 wk & $1.9 \pm 2.3(32)$ & $3.3 \pm 2.2(24)$ & $3.6 \pm 2.5(21)$ & 0.012 & 0.172 \\
\hline $6 \mathrm{mo}$ & $2.1 \pm 2.5(28)$ & $3.1 \pm 2.2(22)$ & $2.9 \pm 2.8(19)$ & 0.251 & 0.629 \\
\hline $1 \mathrm{y}$ & $2.4 \pm 2.3(15)$ & $3.0 \pm 2.9(9)$ & $4.4 \pm 3.5(7)$ & 0.143 & 0.554 \\
\hline \multicolumn{6}{|l|}{ VAS arm } \\
\hline Preoperative & $5.0 \pm 2.6(40)$ & $6.6 \pm 2.2(30)$ & $6.9 \pm 2.6(27)$ & 0.002 & - \\
\hline $6 \mathrm{wk}$ & $2.0 \pm 2.3(38)$ & $3.2 \pm 2.8(30)$ & $3.8 \pm 5.2(24)$ & 0.031 & 0.326 \\
\hline $12 \mathrm{wk}$ & $3.3 \pm 3.7(32)$ & $2.3 \pm 2.4(24)$ & $3.5 \pm 3.4(21)$ & 0.824 & 0.231 \\
\hline $6 \mathrm{mo}$ & $2.9 \pm 3.7(28)$ & $3.1 \pm 2.4(22)$ & $3.3 \pm 3.4(17)$ & 0.693 & 0.991 \\
\hline $1 \mathrm{y}$ & $3.0 \pm 3.0(15)$ & $3.0 \pm 3.3(9)$ & $7.0 \pm 3.8(7)$ & 0.031 & 0.029 \\
\hline \multicolumn{6}{|l|}{ NDI } \\
\hline Preoperative & $25.7 \pm 16.5(40)$ & $41.4 \pm 17.0(30)$ & $50.5 \pm 15.8$ & $<0.001$ & - \\
\hline $6 \mathrm{wk}$ & $23.5 \pm 18.8$ & $35.1 \pm 17.7(30)$ & $35.4 \pm 20.1(24)$ & 0.009 & 0.074 \\
\hline 12 wk & $17.7 \pm 17.2(32)$ & $29.9 \pm 18.1(24)$ & $35.5 \pm 24.5(21)$ & 0.001 & 0.034 \\
\hline $6 \mathrm{mo}$ & $17.7 \pm 20.4(28)$ & $24.0 \pm 14.5(22)$ & $22.2 \pm 23.3(17)$ & 0.388 & 0.755 \\
\hline $1 \mathrm{y}$ & $16.5 \pm 16.3(15)$ & $20.8 \pm 22.8(9)$ & $20.2 \pm 20.7(7)$ & 0.621 & 0.969 \\
\hline \multicolumn{6}{|l|}{ SF-12 PCS } \\
\hline Preoperative & $39.0 \pm 9.1(41)$ & $34.7 \pm 7.4(32)$ & $31.8 \pm 6.7(27)$ & $<0.001$ & - \\
\hline $6 \mathrm{wk}$ & $36.9 \pm 9.3(34)$ & $33.1 \pm 5.3(28)$ & $33.3 \pm 6.8(23)$ & 0.062 & 0.172 \\
\hline $12 \mathrm{wk}$ & $41.2 \pm 10.1(26)$ & $39.1 \pm 7.3(21)$ & $35.0 \pm 8.4(17)$ & 0.019 & 0.216 \\
\hline $6 \mathrm{mo}$ & $43.6 \pm 10.8(24)$ & $40.6 \pm 9.1(20)$ & $36.6 \pm 10.3(12)$ & 0.038 & 0.417 \\
\hline $1 \mathrm{y}$ & $46.4 \pm 8.0(17)$ & $47.6 \pm 7.7(9)$ & $41.2 \pm 15.7(8)$ & 0.254 & 0.524 \\
\hline \multicolumn{6}{|l|}{ 1SF-12 MCS } \\
\hline Preoperative & $55.2 \pm 10.4(41)$ & $45.6 \pm 8.6(32)$ & $33.4 \pm 11.5(27)$ & $<0.001$ & - \\
\hline $6 \mathrm{wk}$ & $55.3 \pm 9.5(34)$ & $49.0 \pm 11.3(28)$ & $43.4 \pm 12.4(23)$ & $<0.001$ & 0.003 \\
\hline 12 wk & $57.4 \pm 8.5(26)$ & $50.6 \pm 9.1(21)$ & $39.6 \pm 13.7(17)$ & $<0.001$ & $<0.001$ \\
\hline $6 \mathrm{mo}$ & $57.8 \pm 7.8(24)$ & $45.1 \pm 10.5(20)$ & $42.7 \pm 14.1(12)$ & $<0.001$ & 0.001 \\
\hline $1 \mathrm{y}$ & $57.0 \pm 8.9(17)$ & $53.8 \pm 5.9(9)$ & $43.9 \pm 16.3(8)$ & 0.006 & 0.025 \\
\hline \multicolumn{6}{|l|}{ PROMIS PF } \\
\hline Preoperative & $42.9 \pm 7.6(42)$ & $37.4 \pm 6.2(32)$ & $35.4 \pm 4.9(27)$ & $<0.001$ & - \\
\hline $6 \mathrm{wk}$ & $43.2 \pm 7.1(33)$ & $38.0 \pm 7.6(23)$ & $41.0 \pm 6.1(17)$ & 0.147 & 0.038 \\
\hline 12 wk & $47.8 \pm 12.1(20)$ & $44.6 \pm 4.7(20)$ & $40.9 \pm 9.5(12)$ & 0.046 & 0.372 \\
\hline $6 \mathrm{mo}$ & $49.3 \pm 8.0(23)$ & $42.5 \pm 9.4(14)$ & $44.5 \pm 8.4(10)$ & 0.070 & 0.145 \\
\hline $1 \mathrm{y}$ & $49.3 \pm 6.1(16)$ & $48.0 \pm 8.3(13)$ & $45.3 \pm 6.1(9)$ & 0.180 & 0.347 \\
\hline
\end{tabular}

Abbreviations: NDI, Neck Disability Index; PROM, patient-reported outcome measure; PROMIS PF, Patient-Reported Outcome Measurement Information System physical function; SF-12 MCS, 2-Item Short Form Mental Composite Summary; SF-12 PCS, 12-Item Short Form Physical Composite Summary; VAS, visual analog scale.

* $P$ values calculated using linear regression to assess outcomes between Patient Health Questionnaire-9) severity groups.

$\dagger P$ values calculated using multiple linear regression to assess the impact of both preoperative Patient Health Questionnaire-9 severity and multilevel procedures on postoperative outcomes.

Boldface indicates significance. 
1-year timepoint $(P=0.029)$. NDI also demonstrated significant differences between groups at the 12-week timepoint $(P=0.034)$ only. Physical function outcomes did not significantly differ as a result of worsening preoperative depression and performing a multilevel procedure at any timepoint except at 6 weeks for PROMIS PF $(P=0.038)$. Performing a multilevel procedure demonstrated significant additive effects on SF-12 MCS for all postoperative timepoints (all $P<0.050$ ). A summary of the added effect of a multilevel procedure with preoperative depression on PROM scores can be found in Table 3.

\section{DISCUSSION}

As the number of mental health studies among spine patients continues to grow, surgeons are becoming more attuned to its impact on postoperative outcomes and a patient's well-being. Although the direct mechanism is still unclear, some studies have suggested that poorer recovery may be a result of decreased motivation or potential dissatisfaction with their overall medical treatment. ${ }^{15,16}$ However, among patients undergoing ACDF, a consensus has yet to be reach regarding depression's impact on outcomes and studies have largely been limited to either analysis at the single level or a mixed single or multilevel cohort. ${ }^{10,11,17,18}$ The current study aimed to provide some clarity on this matter and demonstrated that while preoperative depression still remains a significant risk factor for worse postoperative outcomes, undergoing a multilevel procedure should also be considered a risk factor that exacerbates the negative effects of poorer preoperative mental health.

Among the different timepoints we assessed in the current study, the analysis of preoperative scores demonstrated that baseline pain, disability, and mental and physical health were consistently worse among moderate-to-severe depression groups. Previous studies have established a similar relationship whereby depression, as measured by SF-12 MCS or PHQ-9, was also associated with worse preoperative VAS neck, VAS arm, and NDI. ${ }^{12,18-20}$ Additionally, Blozik et $\mathrm{al}^{21}$ postulated that both depression and anxiety act as determinants of neck pain. Interestingly, few studies have established a similar relationship between preoperative depression severity and physical function using SF-12 $\mathrm{PCS}^{12,20}$ or the Nurick Score, ${ }^{10}$ and even fewer, if any, using PROMIS PF. While our preoperative findings support results from the current literature, this difference between depression severity groups was not maintained through the 1-year timepoint.
Even though baseline arm pain, neck pain, and disability demonstrated differences based on preoperative depression severity, postoperatively, this result did not persist through the 1-year timepoint except for VAS arm. Similar results were also reported by Jenkins et $\mathrm{al}^{20}{ }^{20}$ where the authors demonstrated that differences in preoperative VAS arm and neck, and NDI were not observed at the 1-year timepoint. However, other investigators have reported dissimilar results, with depression severity associated with worse postoperative VAS arm and NDI but not VAS neck. ${ }^{12,18}$ The conflicting results and overall lack of consensus between our study and others may be attributed to a difference in assessment of preoperative depression severity. While our study not only grouped and matched patients into 3 different categories, instead of 2 (depressed vs nondepressed), we also did so using preoperative PHQ-9 rather than SF-12 MCS. Regardless of the psychometric used, collectively our results and others' suggest that patients with increasing depression severity may have an initial delay in improved pain and disability but should be assured that a significantly improved outcome could be attained by 1 -year follow-up.

Preoperative depression severity may also be detrimental to the improvement of physical function. While depression's negative effects on physical function may not be intuitive, a large prospective cohort study ( $n=$ 4757) among general medical practices reported significant associations between lower baseline physical function and depression. ${ }^{22}$ Additionally, the same authors also determined that while the improvement in physical health between nondepressed and depressed individuals did not differ, mean physical function scores at their respective 2-year follow-up were significantly different. The current study evaluated physical function using 2 separate metrics, SF-12 PCS and PROMIS PF, and demonstrated that while preoperative scores may differ between groups, this was not maintained at the 1-year timepoint. A number of other investigators also reported that, within their respective ACDF cohorts, preoperative depression severity was not a significant predictor of physical function. ${ }^{12,18-20}$ However, it should be noted that Phan et $\mathrm{al}^{10}$ reported that increased levels of depression were associated with higher Nurick scores, which serve as a classification system for ambulatory function. These collective results raise the question of whether the underlying reason for depressive symptoms are in fact a "de novo" mental illness or a result of the underlying spinal pathology. In an attempt to address this line of thought, Mangan et al investigated the impact of comorbid depression and/or anxiety on improvements of pain, 
disability, and physical function and demonstrated that no significant associations existed. ${ }^{19}$ Therefore, the results presented in the current study along with those of others suggest that patients undergoing ACDF should expect to achieve a significant improvement in physical function, but may require an extended length of time to reach an appreciable difference.

When considering the potential added risk of a multilevel procedure, the current literature establishes that both single and multilevel ACDF procedures demonstrate significant improvements in postoperative outcomes. However, a number of studies have suggested that multilevel procedures may be associated with longterm adverse events. ${ }^{13,14,23}$ In an effort to evaluate this possibility, we assessed the interaction or collective effect of both preoperative depression and multilevel procedures, which is the main strength of the current study. Few studies have assessed the individual contribution by a number of operative levels and even fewer assessed the collective effect of operative levels and depression severity. Alvin et $\mathrm{al}^{17}$ observed that worse outcomes were attributed to preoperative depression, as evaluated by PHQ-9, but the investigators were unable to establish multilevel procedures as a risk factor. However, the authors of the study assessed the quality of outcomes based on the EuroQol-5D quality of life measure at the 1-year timepoint instead of evaluating more specific metrics individually. Conversely, the current study was able to account for both the number of levels fused among preoperative PHQ-9 matched patients and determined that VAS arm, SF-12 MCS, PROMIS PF, and NDI were affected at intermittent timepoints. This suggests that while it may be prudent for surgeons to consider the impact of both multilevel procedures and degree of depression on a patient's potential outcomes, their combined effect may not be detrimental to postoperative outcomes outside of arm pain and mental health.

The findings of the current study may suggest differences in postoperative recovery with respect to preoperative depressive symptoms following either a single or multilevel ACDF procedure. When considering a cervical fusion procedure, surgeons should be mindful to counsel patients with worse depressive symptoms that following surgery, pain, disability, and physical function will significantly improve but to a limited extent. Both providers and patients should be confident that the addition of another operative level may not be detrimental to the course of postoperative improvement except for mental health outcomes. Therefore, patients presenting with worse depressive symptoms should be advised to seek treatment by a mental health professional prior to or following both single and multilevel ACDF procedures.

\section{Limitations}

This study had a number of limitations that could be addressed in future studies. Patients included in our cohort underwent procedures at a single institution by a sole surgeon, which limits the ability to generalize our results to a much broader population. It is possible that a multicenter study with multiple physicians may provide ample power and allow for a more generalizable result in future studies. Additionally, assessment of all preoperative and postoperative outcomes was conducted through patientreported health questionnaires. This carries potential recall and responder biases and could affect the results of our study. Moreover, our preoperative assessment of depression was not validated by a mental health professional and instead was based on a validated questionnaire for depression screening. Future studies that confirm a diagnosis of depression and/or anxiety may strengthen the results. Lastly, our patient cohort and available data were not conducive to confirming whether treatment of depressive symptoms was sought out prior to or following surgery. Use of therapy, whether cognitive or pharmacologic, could have an impact on reported PROMs and ultimately the combined impact of both depression and multilevel procedures.

\section{CONCLUSION}

Patients with more severe preoperative depressive symptoms demonstrated significantly different baseline values for pain, disability, physical function, and mental health. While patients demonstrated improvements in the postoperative period, disability, arm and neck pain, physical function, and mental health all were intermittently affected by the severity of their respective preoperative depressive symptoms. However, these negative effects on neck pain, disability, and physical function did not persist to the 1-year timepoint. When considering the added effect of a multilevel procedure with severity of preoperative depression, the added numbers of operative levels were not significant contributors to worsened outcomes except for mental health and arm pain. This may suggest that while preoperative depression may significantly impact the course of a patient's postoperative improvement, spine surgeons should be aware of the limited but significant added effect of multilevel procedures. 


\section{REFERENCES}

1. Global, Regional, and National Incidence, Prevalence, and Years Lived with Disability for 354 Diseases and Injuries for 195 Countries and Territories, 1990-2017: a Systematic Analysis for the Global Burden of Disease Study 2017 - The Lancet. https://www. thelancet.com/journals/lancet/article/PIIS0140-6736(18)32279-7/ fulltext

2. Kroenke K, Spitzer RL, Williams JB. The PHQ-9: validity of a brief depression severity measure. J Gen Intern Med. 2001;16(9):606-613. doi:10.1046/j.1525-1497.2001.016009606.x

3. Jenkins NW, Parrish JM, Brundage TS, Hrynewycz NM, Yoo JS, Singh K. Validity of patient health questionnaire-9 in minimally invasive lumbar interbody fusion. Spine. 2020;45(11):E663-E669. doi:10.1097/BRS.0000000000003361

4. Parrish JM, Jenkins NW, Nolte MT, et al. A validation of patient health questionnaire-9 for cervical spine surgery. Spine. 2020;45(23):1668-1675. doi:10.1097/BRS.0000000000003644

5. Tuck AN, Scribani MB, Grainger SD, Johns CA, Knight RQ. The 9-Item Patient Health Questionnaire (PHQ-9): an aid to assessment of patient-reported functional outcomes after spinal surgery. Spine J. 2018;18(8):1398-1405. doi:10.1016/j. spinee.2018.01.004

6. Sundseth J, Kolstad F, Johnsen LG, et al. The Neck Disability Index (NDI) and its correlation with quality of life and mental health measures among patients with single-level cervical disc disease scheduled for surgery. Acta Neurochir (Wien). 2015;157(10):1807-1812. doi:10.1007/s00701-015-2534-1

7. Mathew A, Khan N, Aslam B, Khan MR. Disability and depression in cervical radiculopathy patients: a pilot study. Saudi $J$ Sports Med.2017;17(2):102. doi:10.4103/sjsm.sjsm_57_16

8. Buttermann GR. Anterior cervical discectomy and fusion outcomes over 10 years: a prospective study. Spine. 2018;43(3):207-214. doi:10.1097/BRS.0000000000002273

9. Xie J, Hurlbert RJ. Discectomy versus discectomy with fusion versus discectomy with fusion and instrumentation: a prospective randomized study. Neurosurgery. 2007;61(1):107-116. doi:10.1227/01.neu.0000279730.44016.da

10. Phan K, Moran D, Kostowski T, et al. Relationship between depression and clinical outcome following anterior cervical discectomy and fusion. J Spine Surg. 2017;3(2):133-140. doi:10.21037/ jss.2017.05.02

11. Harris AB, Marrache M, Puvanesarajah V, et al. Are preoperative depression and anxiety associated with patient-reported outcomes, health care payments, and opioid use after anterior discectomy and fusion? Spine J. 2020;20(8):1167-1175. doi:10.1016/j. spinee.2020.03.004

12. Divi SN, Goyal DKC, Mangan JJ, et al. Are outcomes of anterior cervical discectomy and fusion influenced by presurgical depression symptoms on the mental component score of the short form-12 survey? Spine. 2020;45(3):201-207. doi:10.1097/ BRS.0000000000003231

13. Veeravagu A, Cole T, Jiang B, Ratliff JK. Revision rates and complication incidence in single- and multilevel anterior cervical discectomy and fusion procedures: an administrative database study. Spine J. 2014;14(7):1125-1131. doi:10.1016/j.spinee.2013.07.474

14. De la Garza-Ramos R, Xu R, Ramhmdani S, et al. Longterm clinical outcomes following 3- and 4-level anterior cervical discectomy and fusion. J Neurosurg Spine. 2016;24(6):885-891. doi:10.3171/2015.10.SPINE15795
15. Vialle E, de Oliveira Pinto BM, Vialle LR, Gomez JDC. Evaluation of psychosomatic distress and its influence in the outcomes of lumbar fusion procedures for degenerative disorders of the spine. Eur J Orthop Surg Traumatol. 2015;25 Suppl 1:S25-8. doi:10.1007/s00590-015-1641-2

16. Pakarinen M, Vanhanen S, Sinikallio S, et al. Depressive burden is associated with a poorer surgical outcome among lumbar spinal stenosis patients: a 5-year follow-up study. Spine J. 2014;14(10):2392-2396. doi:10.1016/j.spinee.2014.01.047

17. Alvin MD, Miller JA, Lubelski D, et al. The impact of preoperative depression and health state on quality-of-life outcomes after anterior cervical diskectomy and fusion. Global Spine J. 2016;6(4):306-313. doi:10.1055/s-0035-1562932

18. Mayo BC, Massel DH, Bohl DD, et al. Preoperative mental health status may not be predictive of improvements in patientreported outcomes following an anterior cervical discectomy and fusion. J Neurosurg Spine. 2017;26(2):177-182. doi:10.3171/2016. 7.SPINE16472

19. Mangan JJ, Tadley M, Divi SN, et al. The impact of multiple comorbid mental health disorders on health-related quality of life following ACDF. Clin Spine Surg. 2020;33(10):E472-E477. doi:10.1097/BSD.0000000000000957

20. Jenkins NW, Parrish JM, Yoo JS, et al. Are preoperative PHQ-9 scores predictive of postoperative outcomes following anterior cervical discectomy and fusion? Clin Spine Surg. 2020;33(10):E486-E492. doi:10.1097/BSD.0000000000000985

21. Blozik E, Laptinskaya D, Herrmann-Lingen C, et al. Depression and anxiety as major determinants of neck pain: a crosssectional study in general practice. BMC Musculoskelet Disord. 2009;10:13. doi:10.1186/1471-2474-10-13

22. Stegenga BT, Nazareth I, Torres-González F, et al. Depression, anxiety and physical function: exploring the strength of causality. J Epidemiol Community Health. 2012;66(7):e25. doi:10.1136/ jech.2010.128371

23. Gornet MF, Lanman TH, Burkus JK, et al. One-level versus 2-level treatment with cervical disc arthroplasty or fusion: outcomes up to 7 years. Int J Spine Surg. 2019;13(6):551-560. doi:10.14444/6076

Funding: No funds were received in support of this work.

Declaration of Conflicting Interests: The authors report no conflicts of interest related to this work.

\section{IRB Approval: ORA \#14051301}

Corresponding Author: Kern Singh, Department of Orthopaedic Surgery, Rush University Medical Center, 1611 W Harrison St, Suite \#300, Chicago, IL 60612, USA; kern.singh@ rushortho.com

Published 15 February 2022

This manuscript is generously published free of charge by ISASS, the International Society for the Advancement of Spine Surgery. Copyright (C) 2022 ISASS. To see more or order reprints or permissions, see http:// ijssurgery.com. 